\title{
Therapeutic drug monitoring in patients with cystic fibrosis and mycobacterial disease
}

\author{
M. Gilljam*\#, S.E. Berning**, C.A. Peloquin**, B. Strandvik" ${ }^{\# *}$, L.O. Larsson*
}

Therapeutic drug monitoring in patients with cystic fibrosis and mycobacterial disease. $M$. Gilljam, S.E. Berning, C.A. Peloquin, B. Strandvik, L.O. Larsson. (C)ERS Journals Ltd 1999.

ABSTRACT: Cystic fibrosis (CF) patients require higher dosages of many antibiotics. The relapse of tuberculosis in one $\mathrm{CF}$ patient, and the repeated growth of Mycobacterium avium-intracellulare in another, despite conventional therapy, raised the question of whether the serum levels of the antimycobacterial drugs were adequate.

Antimycobacterial drug serum concentrations were assayed in $10 \mathrm{CF}$ patients with pulmonary mycobacterial disease.

Serum levels below the proposed target range were seen $2 \mathrm{~h}$ after drug intake in the initial four patients treated: for rifampicin in $2 / 3$, ethambutol in $3 / 4$ and for clarithromycin in $2 / 3$ patients, despite standard dosages. Reassays after dose adjustment and assays in six other patients showed that adequate levels were not achieved $4 \mathrm{~h}$ after chlaritromycin in $3 / 5$, ethambutol in $1 / 5$, ciproflaxacin in $1 / 2$ and ofloxacin in $2 / 2$ patients. The patient with relapse of tuberculosis and the patient with continuous growth of $M$. avium-intracellulare improved and became culture negative after dose adjustment.

Low drug serum levels is one reason for therapy failure in cystic fibrosis patients with mycobacterial disease. Therapeutic drug monitoring is recommended.

Eur Respir J 1999; 14: 347-351.
*Dept of Respiratory Medicine and Allergology, ${ }^{\sharp}$ Dept of Paediatrics, and ${ }^{\#}$ Gothenburg Cystic Fibrosis Center, Göteborg University, Sweden **National Jewish Medical and Research Center, Denver, CO, USA.

Correspondence: M. Gilljam, Dept of Respiratory Medicine and Allergology, Sahlgrenska University Hospital, S-413 45, Göteborg, Sweden. Fax: 4631824904

Keywords: Cystic fibrosis mycobacterial disease therapeutic drug monitoring

\section{Received: November 31998}

Accepted after revision March 281999

This work was supported by the Swedish Heart and Lung Foundation and the Märta and Gunnar Bergendahls Foundation.This work was presented in part at the American Thoracic Society annual meeting in San Francisco, May 1997.
Cystic fibrosis $(\mathrm{CF})$ is the most common lethal inherited disease in Caucasians, but the survival rate has steadily improved [1]. Recurrent respiratory infections leading to destruction of the lungs and respiratory insufficiency are still the major causes of death. Mycobacterial pulmonary disease has been considered uncommon in CF. However, some reports [2-5] indicate that growth of mycobacteria in the sputum of CF patients is not unusual. The relapse of pulmonary tuberculosis in one patient and the repeated growth of Mycobacterium avium-intracellulare in another CF patient despite treatment for several months, raised the question of whether the recommended dosages used were sufficient. The renal and nonrenal clearance for most, but not all, drugs is enhanced in CF. Pharmacokinetic studies of antibiotics have, however, shown contradictory results [6]. Therapeutic drug monitoring has been shown to be useful in patients with multiresistant tuberculosis and disease caused by M. avium-intracellulare [7]. As a part of the clinical assessment, serum concentrations of antimycobacterial drugs were assayed in $10 \mathrm{CF}$ patients during treatment for mycobacterial pulmonary disease. Four patients were reassayed after dose adjustment.

\section{Material and methods}

\section{Study population}

One patient with pulmonary tuberculosis (patient 1) and nine patients (patients $2-10$ ) with pulmonary disease com- plicated by nontuberculous mycobacteria (NTM) are reported. Patient 1, a female with moderate CF [8] was bacille Calmette-Guérin (BCG) vaccinated at birth (table 1). Pulmonary tuberculosis was diagnosed at $25 \mathrm{yrs}$ of age. M. tuberculosis was grown in sputum culture, although acid-fast bacilli (AFB) smear test was negative. The purified protein derivative (PPD) tuberculin skin test was $13 \mathrm{~mm}$. The patient was treated with isoniazid (INH), ethambutol (EMB), pyrazinamide (PZA) and, initially, rifampicin (RMP). Because of adverse effects, RMP was changed to streptomycin. Later, a combination of rifabutin (RBT), cycloserine, EMB and INH was used. The treatment was extended to 30 months with a combination of 2-4 drugs.

Five years later, the patient became pregnant after in vitro fertilization. At 18 weeks gestation, the patient presented with a 10-week history of increased asthmatic symptoms, dyspnoea, production of sputa and also emesis. Because of heavy growth of Pseudomonas aeruginosa in sputum, intravenous treatment with ceftazidime and tobramycin was started and the patient's condition improved. The initial smear was negative for AFB, but culture was positive for M. tuberculosis, with full susceptibility, after 8 weeks of incubation. Polymerase chain reaction (PCR) of sputum for $M$. tuberculosis had previously been negative, but became positive at this point. At the time of relapse of tuberculosis, the patient had a forced expiratory volume in one second (FEV1) of $60 \%$ of predicted. Treatment with INH, RMP and PZA was started. According to 
Table 1. - Characteristics of patients at the time therapy for mycobacterial disease was initiated

\begin{tabular}{|c|c|c|c|c|c|c|c|c|c|}
\hline $\begin{array}{l}\text { Patient } \\
\text { No. }\end{array}$ & $\begin{array}{l}\text { Age } \\
\text { yrs }\end{array}$ & Sex & $\mathrm{PI} / \mathrm{PS}$ & Genotype & $\begin{array}{l}\text { FEV1 } \\
\% \text { pred }\end{array}$ & $\begin{array}{c}\mathrm{BMI} \\
\mathrm{kg} \cdot \mathrm{m}^{-2}\end{array}$ & $\begin{array}{c}\text { Other } \\
\text { features }\end{array}$ & $\begin{array}{l}\text { Sputum } \\
\text { cultures }\end{array}$ & Mycobacteria \\
\hline 1 & 32 & $\mathrm{~F}$ & PI & $\Delta \mathrm{F} 508 / ?$ & 60 & 22 & EFAD & PA & M. tuberculosis \\
\hline 2 & 20 & M & PI & $\Delta \mathrm{F} 508 / \Delta \mathrm{F} 508$ & 55 & 17 & EFAD & $\mathrm{SA}, \mathrm{PA}, \mathrm{AF}$ & M. avium-intracellulare* \\
\hline 3 & 20 & $\mathrm{~F}$ & PI & $\Delta \mathrm{F} 508 / \Delta \mathrm{F} 508$ & 30 & 16 & EFAD, LD & SA, PA & M. avium-intracellulare \\
\hline 4 & 21 & $\mathrm{~F}$ & PI & $\Delta \mathrm{F} 508 / \mathrm{E} 60 \mathrm{X}$ & 80 & 23 & & $\mathrm{SA}, \mathrm{PA}$ & M. avium-intracellulare* \\
\hline 5 & 27 & M & PI & $\Delta \mathrm{F} 508 / \Delta \mathrm{F} 508$ & 80 & 22 & & SM & M. avium-intracellulare* \\
\hline 6 & 25 & $\mathrm{~F}$ & PI & $\Delta \mathrm{F} 508 / 621+1 \mathrm{G}-\mathrm{T}$ & 78 & 20 & EFAD & SA & M. avium-intracellulare* \\
\hline 7 & 31 & $\mathrm{M}$ & PI & $\Delta \mathrm{F} 508 / \Delta \mathrm{F} 508$ & 80 & 22 & LD, DM & SM, SA & M. avium-intracellulare \\
\hline 8 & 17 & $\mathrm{~F}$ & PI & $\Delta \mathrm{F} 508 / \Delta \mathrm{F} 508$ & 117 & 20 & & $\mathrm{BC}, \mathrm{AF}$ & M. abscessus* \\
\hline 9 & 22 & $\mathrm{~F}$ & PS & V392G/? & 60 & 19 & & PA & M. abscessus* \\
\hline 10 & 32 & $\mathrm{~F}$ & PS & $\mathrm{R} 1066 \mathrm{C} / 3849+10 \mathrm{kbG}-\mathrm{T}$ & 65 & 22 & & PA & M. abscessus* \\
\hline
\end{tabular}

PI: pancreatic insufficiency, requiring enzymes; PS: pancreatic sufficiency; ?: unknown mutation; FEV1: forced expiratory volume in one second, \% of predicted; BMI: body mass index; EFAD: essential fatty acid deficiency; LD: biopsy-verified mild cystic fibrosis (CF) related liver disease; DM: cystic fibrosis related diabetes mellitus requiring insulin; PA: Pseudomonas aeruginosa; SA: Staphylococcus aureus; AF: Aspergillus fumigatus; SM: Stenotrophomonas maltophilia; BC: Burkholderia cepacia; M.: mycobacterium; M: male; F: female. *: Positive smear test.

local guidelines, and after review of the literature, standard treatment was chosen despite the patient being pregnant [9]. After almost 2 months of treatment and after 35 weeks of gestation, the patient gave birth to a healthy child. However, the patient did not improve as expected during treatment, and assays for drug serum concentrations were performed.

The characteristics of all patients studied are given in table 1. All patients had a decline in FEV1 and/or symptoms such as weight loss, increased sputum production, night sweat and haemoptysis, and without the usual effects of intravenous antibiotics against other bacteria cultivated from sputum. Patient 2 had continuous growth of $M$. avium-intracellulare in sputum despite several months of treatment with EMB, RMP and clarithromycin and therefore RMP was changed to RBT. Patient 3 had treatment with two intravenous antipseudomonal antibiotics at the time of the assays. Patient 9 was also smear positive and with symptoms of infection after $1 \mathrm{yr}$ of treatment. Patient 10 had initially been treated with amikacin intravenously for 6 weeks. All patients were monitored for possible side effects from antimycobacterial medication according to local guidelines.

Table 2. - Drugs used in the study and total daily dose in each patient

\begin{tabular}{lrrrrrrrrr}
\hline & \multicolumn{10}{c}{ Patient } \\
\cline { 2 - 9 } Drug & $1 *$ & $2 *$ & $3 *$ & 5 & 6 & 7 & 8 & 9 & 10 \\
\hline Isoniazid & 7 & & & & & & & & \\
Rifampicin & 14 & & 16 & & 11 & 9 & 11 & & \\
Ethambutol & & 27 & 32 & 18 & & 19 & 16 & & \\
Clarithromycin & & 14 & 20 & & & 16 & & 18 & 16 \\
Rifabutin & & 6 & & 4 & & & & & \\
Ofloxacin & & & & & 16 & & 11 & & \\
Ciprofloxacin & & & & & & & & 27 & 23 \\
\hline
\end{tabular}

Drugs are presented as $\mathrm{mg} \cdot \mathrm{kg}$ body weight ${ }^{-1} \cdot \mathrm{day}^{-1} \cdot *$ : Total daily dose after adjustment. Ofloxacin and ciprofloxacin were administered in two equal doses. In patients 7, 9 and 10, clarithromycin was administered in two equal doses; in patients 2 and 3, 500 $\mathrm{mg}$ was administered in the morning and $250 \mathrm{mg}$ in the evening. All other drugs were administered as a single daily dose.

\section{Drug monitoring}

Eight different drugs were assessed (table 2). For reviews of the pharmacokinetic characteristics of the drugs, see Peloquin [7, 10].

The antimycobacterial drugs were excluded the day before patients 1-4 were admitted for assays and reassays. In patients $5-10$, all drugs were taken on an ordinary steady state regimen and without pancreatic enzymes. The patients were fasting from midnight until the 2-h blood samples were drawn. A nurse supervised the antimycobacterial drug administration and collected blood by direct venipuncture. In the first assay of patients $1-4$, blood samples were only drawn at $2 \mathrm{~h}$ postdrug intake, a time which should reflect peak concentrations for most drugs, specifically if the drugs were taken on an empty stomach. After having received results of low levels for several drugs, subsequent samples were collected both 2 and $4 \mathrm{~h}$ after drug intake to better evaluate the absorption pattern. Reassays from patient 4 were unfortunately lost during transportation.

The samples were centrifuged at room temperature for 10 minutes at $4 \times g$ and the serum collected in polypropylene tubes and stored frozen at $-70^{\circ} \mathrm{C}$. All analyses were performed with high-performance liquid chromatography (HPLC) and gas-liquid chromatography (GLC) [10] at the National Jewish Medical and Research Center, Denver, CO, USA.

\section{Results}

In the initial assay, low serum concentrations were observed $2 \mathrm{~h}$ after drug intake in the patient with pulmonary tuberculosis (patient 1) for INH and RMP, but adequate for PZA $\left(1.96,0.17\right.$ and $42.51 \mu \mathrm{g} \cdot \mathrm{mL}^{-1}$ respectively), when compared to the proposed target ranges for mycobacterial disease $\left(3-5,8-24\right.$, and $20-60 \mu \mathrm{g} \cdot \mathrm{mL}^{-1}$ respectively) [10]. After dose adjustment the levels were within the proposed target range at $2 \mathrm{~h}$ for INH and $4 \mathrm{~h}$ for RMP (table 3 ). Patient 1 improved after dose adjustment, and before the treatment was terminated a bronchoscopy with bronchoalveolar lavage (BAL) was performed. Patient 1 was culture negative for mycobacteria in BAL. The patient 
Table 3. - Drug serum concentrations in patients 1-3 (after reassay) and patients 5-10 (first assay)

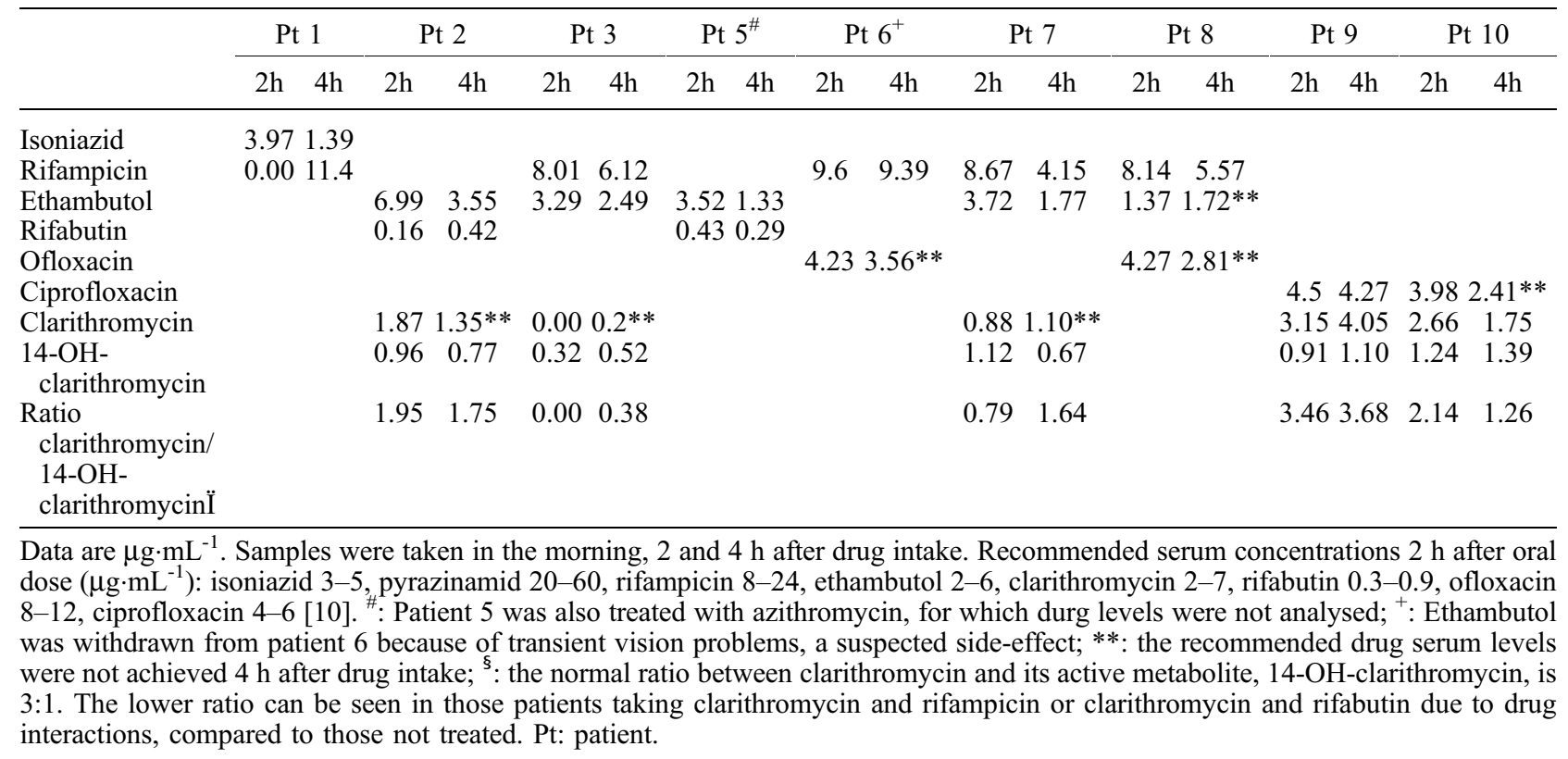

was treated with PZA for 2 months according to the recommended regime, while RMP and INH were extended to 16 months.

For the initial assays of patients 2-4, the levels were below the target range for RMP in patient $3\left(2.24 \mu \mathrm{g} \cdot \mathrm{mL}^{-1}\right)$, for clarithromycin in patients 3 and 4 (1.22 and 0.53 $\mu \mathrm{g} \cdot \mathrm{mL}^{-1}$, respectively) and for EMB in patients $2-4(0.03$, 1.2 and $1.78 \mu \mathrm{g} \cdot \mathrm{mL}^{-1}$, respectively) $2 \mathrm{~h}$ post dose (proposed target ranges $8-24,2-7$ and $2-6 \mu \mathrm{g} \cdot \mathrm{mL}^{-1}$, respectively for each drug). The dosages given for patients 2-4 (in $\mathrm{mg} \cdot \mathrm{kg}$ body weight ${ }^{-1}$ ) were: RMP 10,8 and 10 EMB 24, 26 and 19; and clarithromycin 10, 13 and 16.

The dosages given and the results of the reassays after dose adjustment in patients 1-3 and the assays in patients 5-10, 2 and $4 \mathrm{~h}$ after drug intake are depicted in tables 2 and 3. Patient 2 had a delayed but adequate absorption of rifabutin in the second assay. The levels of EMB had risen immensely. The sputum culture became negative 3 months after the change of antibiotics and dose adjustment. Patient 2 also gained weight after gastrostomy for nutritious support. The patient's slow decline in FEV1 ceased during treatment. Patient 3 had advanced disease, was accepted for lung transplantation, but unfortunately died during the operation. The patient was culture negative for mycobacteria in repeated sputum samples during treatment, and no mycobacteria were grown from lung tissue post mortem. Patient 4 improved and stopped treatment after 22 months. At this time the patient's liver enzymes had risen slowly, but normalized after withdrawal of the medication.

Serum levels were in the lower range for all RMP patients in the reassays, after dose adjustment in patients 13 and the initial assays in patients $5-10$. Proposed target ranges were not achieved $4 \mathrm{~h}$ after drug intake in $3 / 5$ patients for clarithromycin, 1/2 for ciprofloxacin, 1/5 for EMB and $2 / 2$ patients for oxfloxacin.

Patients 6-9 are currently stable, although patients 8-9 still have growth of Mycobacterium abcsessus in the sputum. Patient 10 improved soon after start of therapy, and their FEV1 is now back to $90 \%$ pred, and sputum cultures are negative for $M$. abcsessus.

\section{Discussion}

This study found low serum levels for several drugs in two patients with therapy failure despite conventional treatment for pulmonary disease caused by $M$. tuberculosis and $M$. avium-intracellulare. Dose adjustment resulted in a clinical improvement. Low serum levels were seen for several drugs in eight other $\mathrm{CF}$ patients treated for mycobacterial disease. Disease owing to $M$. tuberculosis, an obligate pathogen, is usually easier to treat than NTM, which is more difficult to differentiate between colonization, infection and disease [11-13]. Symptoms indicative of mycobacterial disease can be consistent with infectious exacerbation of $P$. aeruginosa in CF patients and it is also difficult to interpret radiographic changes. If growth of NTM is related to clinical and/or pulmonary deterioration, treatment usually results in an improvement of pulmonary function [3]. It has been suggested that treatment should focus on clinical improvement and not eradication [14], but in patients with CF eradication would be preferable. On the contrary, M. tuberculosis should be "treated promptly", even if there is only sparse growth in one culture. The competence of the microbiological laboratory is of importance, and specific pretreatment of the CF sputum is necessary [15]. The use of PCR in the diagnosis of mycobacterial disease is controversial but would be valuable considering the difficulty of culturing $\mathrm{CF}$ sputum. There is, however, a risk of both false positive and false negative results of PCR [16], the latter occurring initially in case 1 . Skin tests for sensitins and tuberculin are reported to be of limited diagnostic value [3, 17].

Interactions between the multiple medications, pancreatic insufficiency, disturbed gastrointestinal motility and increased metabolic rate because of increased renal and nonrenal clearance [6] are all factors leading to the difficulty in achieving adequate drug serum concentrations 
in CF. The nutritional status of the patient is important for the overall outcome in CF [18]; and side effects from the antimycobacterial drugs, including emesis, can cause difficulties in the management of the therapy.

Patient 1 had pulmonary tuberculosis, and treatment was mandatory. It was puzzling that the patient had a relapse of tuberculosis after such long-lasting previous treatment, and with three or four antibiotics all the time (the patient has previously been reported in a study by HJELTE et al. [3]). Unfortunately, corresponding DNA fingerprinting by restriction fragment length polymorphism [19] could not be obtained in this study, since the original strain was not saved. The patient has had no known infectious tuberculous contact and was therefore considered to have had a relapse. The first assay showed low serum levels $2 \mathrm{~h}$ after administration of INH and RMP, the drugs considered as first-line therapy, but adequate levels at reassay and after dose adjustment, despite delayed absorption of RMP. The patient did not have RMP during the first treatment period 5 yrs previously, but had been given streptomycin, another bactericidal drug. The dosages during the first treatment period were similar to those at the beginning of the relapse of tuberculosis. The first treatment may not have completely eradicated the bacteria due to low serum concentrations. Altered pharmacokinetics during pregnancy may explain the poor initial therapy results but not the low drug serum levels after birth [20].

Patient 4 improved, perhaps because of adequate serum concentrations of RMP, a bactericidal drug, and a good clinical and nutritional condition. In patient 2, the EMB concentration was almost undetectable in the first assay, but had, after adjustment, risen to the upper recommended limit. The increase in dosage could not explain the high increase of serum EMB level. Uneven and/or delayed absorption in the initial assay may be one explanation. Interactions between clarithromycin and RBT may explain the low serum level of clarithromycin in the reassay despite higher dosage [21]. Clarithromycin is known to reach high concentrations in phagocytes and tissues [22] and since the authors were concerned about the risk of sideeffects the dosage was not increased further. Patient 3 had advanced CF with a low body mass-index (BMI). Altered volume of distribution and upregulated liver enzymes by RMP may explain the low serum drug levels despite the high dosages per kilogram of body weight used.

The pancreatic status and disturbance in gastrointestinal motility could explain low serum drug levels after oral intake. However, a low level of ciprofloxacin was found in one pancreatic sufficient patient. Acquired drug resistance and disease relapse has been reported for patients with malabsorption [23-25]. Only one of the three patients in the current study with growth of $M$. abscessus had negative sputum cultures one year after the start of therapy. $M$. abscessus is known to be difficult to treat and it can only be speculated as to whether the initial treatment with amikacin and rapid dose adjustment contributed to the result.

Since this study was undertaken, guidelines have been published for the management of NTM disease in CF [26]. Compared to the directions given for drugs and dosages, the patients in the present study had a lower dosage for clarithromycin. In the first assays, the patients were not in a complete steady-state since the doses the day before sampling were omitted, but it is not believed that the result would have differed much in complete steady-state since most of the drugs were at $75-90 \%$ of the steadystate levels at the times of the assays even after temporary discontinuation [27]. However, HPLC and GLC have the advantage over biological methods in that they allow the simultaneous assessment of all drugs without the need to cease treatment [10], and it is recommended that the assays are performed in the steady-state.

In conclusion, mycobacterial disease can be an important factor contributing to the deterioration of pulmonary function in cystic fibrosis. Mycobacterium tuberculosis is an obligate pathogen, and the decision to treat pulmonary infection or disease caused by nontuberculous mycobacteria has to be assessed for the individual patient. Subtherapeutic serum drug levels is one reason for therapy failure. Considering the results of this study and previous studies in patients with acquired immunodeficiency syndrome, the authors suggest that therapeutic drug monitoring is performed after a few weeks of treatment and that samples are taken in steady-state, at 2, 4 and, if possible, 6 $\mathrm{h}$ after drug intake. The number of cystic fibrosis patients requiring treatment for mycobacterial disease is low and a prospective multicentre study would be necessary to answer the question of dosage recommendations in order to achieve therapeutic drug serum levels. Rifampicin, ethambutol and clarithromycin would be of interest to assay because of their central role in the treatment of nontuberculous mycobacteria and their pharmacological profile including the risk of malabsorption. Clinical follow-up and bronchoscopy with bronchoalveolar lavage after treatment would help to address the issue of therapy effect and eradication of mycobacteria.

Acknowledgements. Patients 9 and 10 attended the Adult Cystic Fibrosis Clinic, St Michael's Hospital, Toronto, Canada. The authors thank E. Tullis for contributing with these patients and for valuable help with the manuscript.

\section{References}

1. Lewis PA. The epidemiology of cystic fibrosis. In: Hodson ME, Geddes DM, eds. Cystic Fibrosis. London, Chapman and Hall, 1995; pp. 1-13.

2. Smith M, Efthimiou J, Hodson M, Batten J. Mycobacterial isolations in young adults with cystic fibrosis. Thorax 1984; 39: 369-375.

3. Hjelte L, Petrini B, Källenius G, Strandvik B. Prospective study of mycobacterial infections in patients with cystic fibrosis. Thorax 1990; 45: 397-400.

4. Kilby J, Gilligan P, Yankaskas J, Highsmith W, Edwards L, Knowles M. Nontuberculous mycobacteria in adult patients with cystic fibrosis. Chest 1992; 102: 70-75.

5. Aitken M, Burke W, McDonald G, Wallis C, Ramsey B, Nolan C. Nontuberculous mycobacterial disease in adult cystic fibrosis patients. Chest 1993; 103: 1096-1099.

6. Kavanagh RE, Unadkat JD, Smith AL. Drug disposition in cystic fibrosis. In: Davies PB, ed. Cystic Fibrosis, Vol. 64. New York, Marcel Dekker, 1993; pp. 91-136.

7. Peloquin CA. Using therapeutic drug monitoring to dose the antimycobacterial drugs. Clin Chest Med 1997; 18: 79-87.

8. Schwachman H, Kulczycki L. Long-term study of one hundred five patients with cystic fibrosis. Am J Dis Child 1958; 96: 6-15. 
9. Davidson P. Managing tuberculosis during pregnancy. Lancet 1995; 346: 199-200.

10. Peloquin CA. Therapeutic drug monitoring of the antimycobacterial drugs. Clin Lab Med 1996; 16: 717-729.

11. Kinney J, Little B, Yolken R, Rosenstein B. Mycobacterum avium complex in a patient with cystic fibrosis: disease versus colonization. Pediatr Infect Dis J 1989; 8: 393-396.

12. Griffith D, Girard W, Wallace R Jr. Clinical features of pulmonary disease caused by rapidly growing mycobacteria. Am J Respir Dis 1993; 147: 1271-1278.

13. Wallace R Jr, Glassroth J, Griffith D, Olivier N, Cook J, Gordin F. Diagnosis and treatment of disease caused by nontuberculous mycobacteria. Am J Respir Crit Care Med 1997; 156 (Suppl. 2): s1-s25.

14. Hjelt K, Højlyng N, Howitz P, et al. The role of mycobacteria other than tuberculosis (MOTT) in patients with cystic fibrosis. Scand J Infect Dis 1994; 26: 569-576.

15. Whittier S, Olivier K, Gilligan P, Knowles M, Della-Latta P. Proficiency testing of clinical microbiology laboratories using modified decontamination procedures for detection of nontuberculous mycobacteria in sputum samples from cystic fibrosis patients. J Clin Microbiol 1997; 35: 2706-2708.

16. Soini H, Viljanen M. Gene amplification in the diagnosis of mycobacterial infections. APMIS 1997; 105: 345-353.

17. Mulherin D, Coffey M, Halloran D, Keogan M, Fitzgerald M. Skin reactivity to atypical mycobacteria in cystic fibrosis. Respir Med 1990; 84: 273-276.
18. Durie PR, Pencharz PB. Cystic fibrosis: nutrition. Br Med Bull 1992; 48: 823-846.

19. van Soolingen D, Hermans P. Epidemiology of tuberculosis by DNA fingerprinting. Eur Respir J 1995; 8: Suppl. 20, 640s-656s.

20. Hedstrom S, Martens M. Antibiotics in pregnancy. Clin Obstet Gynecol 1993; 36: 886-892.

21. Peloquin C, Berning S. Evaluation of the drug interaction between clarithromycin and rifampin. J Infect Dis Pharmacother 1996; 2: 19-35.

22. Sturgill M, Rapp R. Clarithromycin: review of a new macrolide antibiotic with improved microbiologic spectrum and favorable pharmacokinetic and adverse effect profiles. Ann Pharmacother 1992; 26: 1099-1108.

23. Turner M, McGowan C, Nardell E, Haskal R. Serum drug levels in tuberculosis patients. Am J Respir Crit Care Med 1994; 149: A527.

24. Patel K, Belmonte R, Crowe H. Drug malabsorption and resistant tuberculosis in HIV-infected patients. $N$ Engl $J$ Med 1995; 332: 336-337.

25. Peloquin C, Nitta A, Burman W, et al. Low antituberculosis drug concentrations in patients with AIDS. Ann Pharmacother 1996; 30: 919-925.

26. Olivier K, Yankaskas J, Knowles M. Nontuberculous mycobacterial disease in cystic fibrosis. Semin Respir Infect 1996; 11: 272-284.

27. Winter ME. In: Young LY, Koda-Kimble MA. Basic Clinical Pharmacokinetics eds. 2nd Edn. Applied Therapeutics, Washington, Spokane, 1988; pp. 1-14. 